\title{
The influence of bone substitute materials on the bone volume after maxillary sinus augmentation: a microcomputerized tomography study
}

\author{
Sebastian Kühl • Christoph Brochhausen • \\ Hermann Götz • Andreas Filippi • Michael Payer • \\ Bernd d'Hoedt • Matthias Kreisler
}

Received: 13 October 2011 /Accepted: 9 April 2012 /Published online: 27 April 2012

(C) Springer-Verlag 2012

\begin{abstract}
Objectives This study aims to evaluate the effect of adding bone substitute materials (BSM) to particulated autogenous bone $(\mathrm{PAB})$ on the volume fraction (Vf) of newly formed bone after maxillary sinus augmentation.

Materials and methods Thirty healthy patients undergoing maxillary sinus augmentation were included. PAB $(N=10)$, mixtures of $\mathrm{PAB}$ and beta-tricalciumphosphate $(\mathrm{PAB} / \beta-\mathrm{TCP})$
\end{abstract}

S. Kühl $(\bowtie) \cdot$ A. Filippi

School of Dental Medicine, Department of Oral Surgery,

Oral Radiology and Oral Medicine, University of Basel,

Hebelstrasse 3,

4056 Basel, Switzerland

e-mail: sebastian.kuehl@unibas.ch

C. Brochhausen

REPAIR-lab, Institute of Pathology, University Medical Center of the Johannes Gutenberg University Mainz,

Langenbeckstrasse 1,

55131 Mainz, Germany

H. Götz

Institute for Applied Structure and Microanalysis, University

Medical Center of the Johannes Gutenberg University Mainz,

Obere Zahlbacherstr. 63,

55131 Mainz, Germany

\section{Payer}

Department of oral Surgery and Radiology,

School of Dentistry, Medical University of Graz, Austria,

Auenbruggerplatz 12,

8063 Graz, Austria

B. d'Hoedt • M. Kreisler

School of Dental Medicine; Department of Oral Surgery,

University Medical Center of the Johannes Gutenberg University

Mainz,

Augustusplatz 2,

55131 Mainz, Germany
$(N=10)$, as well as PAB and $\beta$-TCP and hydroxyapatite $(\mathrm{PAB} / \mathrm{HA} / \beta-\mathrm{TCP})(N=10)$ were randomly used for sinus augmentation. A sample of the graft material was maintained from each patient at time of maxillary sinus augmentation, and Vfs of the PAB and/or BSM in the samples were determined by means of microcomputerized tomography $(\mu-\mathrm{CT})$. Five months later, samples of the grafted areas were harvested during implantation using a trephine bur. $\mu-C T$ analysis of these samples was performed, and the Vf of bone and BSM were compared with the data obtained 5 months earlier from the original material.

Results The mean Vf of the bone showed a statistically significant increase $(p<0.05)$ in all groups after a healing period of 5 months without statistically significant difference between the groups.

Conclusions With regard to the increase of bone volume, it is not relevant if PAB is used alone or combined with $\beta$-TCP or HA $/ \beta$-TCP.

Clinical relevance The amount of $\mathrm{PAB}$ and associated donor site morbidity may be reduced by adding BSM for maxillary sinus augmentation.

Keywords Maxillary sinus augmentation $\cdot$ Bone $\cdot$ Substitute materials

\section{Introduction}

The severely atrophic posterior maxilla can successfully be treated by maxillary sinus augmentation as described by Boyne and James or Tatum [1-6] prior to implant placement. Though many studies have documented the suitability of different materials for maxillary sinus augmentation, such 
as autogenous bone, biomaterials, or combinations of both [7-13], it still remains widely unknown which material is the most favorable [14-16].

Regardless of the material used for augmentation, the structure of the graft after healing must provide a microarchitecture that allows a sufficient implant anchorage through osseointegration. The mechanical stability of osseointegrated implants is dependent on the quality and quantity of the adjacent bone and its biomechanical properties. Therefore, obtaining a maximum of bone quantity is one of the key goals in augmentative implant surgery.

Many studies have evaluated the amount of newly formed bone following maxillary sinus augmentation with different grafting materials in histological sections of samples retrieved several months after lateral antrostomy [14, 17-22]. Though autogenous bone is still regarded as standard for grafting [23, 24], it could be shown that a remarkable amount of newly formed bone is obtainable when substitute materials are used alone $[18,25]$. Since it is well known that autogenous bone has osteoinductive properties [26, 27], the question arises as to whether mixtures of bone and BSM result in a similar amount of newly formed bone when used for maxillary sinus augmentation as for autogenous bone alone.

When adding autogenous bone to substitute materials for maxillary sinus augmentation, the correct determination of the amount of newly formed bone in histological samples presents a challenge because the volumetric distribution and amount of bone and substitute material at the time of maxillary sinus augmentation is unknown. $\mu$-CT was successfully used to visualize and evaluate the volumetric distribution of bone and substitute materials in bony samples retrieved after maxillary sinus augmentation [28]. As a non-invasive technique, $\mu$-CT might also be suitable to evaluate the volumetric distribution of bone and BSM in a particulated form, e.g., the particulated graft at the time of maxillary sinus augmentation. Comparing the volumetric distributions of bone and $\mathrm{BSM}$ at the time of sinus augmentation with the volumetric distributions in samples retrieved several months after healing has not been performed until now. This allows the determination of changes in the volumes of bone and substitute material over time. The working hypothesis is that adding BSM to PAB results in a smaller amount of newly formed bone volume 5 months after maxillary sinus augmentation when compared to the use of PAB alone.

\section{Materials and methods}

The study protocol was approved by the ethics committee of the Johannes Gutenberg University of Mainz (No. 837.158.08 (6155)).

\section{Patients}

Thirty consecutive, systemically healthy patients (17 female, 13 male; mean age 54; range 36-79) who presented for maxillary sinus augmentation were included in this study. Informed consent was obtained from all patients prior participation in this study. Exclusion criteria were advanced systemic diseases, osteoporosis, pregnancy, infectious diseases, radiation, corticosteroid and bisphosphonate medication, chemotherapy, and smoking.

Before surgical treatment, patients were randomly enrolled in one of the following treatment groups:

Group A: Mixture of blood, particulated autogenous bone (PAB), and $\beta$-TCP $(N=10)$.

Group B: $\quad$ Mixture of blood, PAB, and $\beta-\mathrm{TCP} / \mathrm{HA}(N=10)$. Group C: Mixture of blood and PAB $(N=10)$.

Surgical procedure

A mucoperiosteal flap was elevated and access to the sinus membrane was achieved by drilling an oval hollow into the facial sinus wall with a diamond bur. The bone in the center of the drilled window was gently infractured and the intact Schneiderian membrane was elevated. Once the Schneiderian membrane was completely mobilized, the infractured bone was folded inwards and upwards, imitating a trapdoor effect.

Autogenous bone was harvested as a block either from the chin or the linea obliqua externa and particulated using a surgical bone mill (Quetin bone mill ${ }^{\circledR}$, Quetin Dental Products, Leimen, Germany). The particulated bone was mixed with blood, and BSM was added in a ratio of

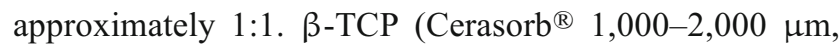
Curasan AG, Kleinostheim, Germany) was used in group A $(N=10)$ and a $\beta$-TCP/HA mixture (Straumann ${ }^{\circledR}$ BoneCeramic 500-1,000 $\mu \mathrm{m}$; Institut Straumann AG, Basel, Switzerland) in group B $(N=10)$. In group C $(N=10)$, only $\mathrm{PAB}$ and blood were used without any additional BSM.

A sample of approximately $0.5 \mathrm{~cm}^{3}$ of each particulated graft was collected at the time of sinus augmentation and maintained in a standard polypropylene snap-lock tube (Eppendorf Safe-Lock Tubes ${ }^{\circledR} 0.5 \mathrm{ml}$, Eppendorf AG, Hamburg, Germany). For plugging the graft into the tubes, the same forces as for the maxillary sinus augmentation were applied as far as possible. They were plugged by one of the two oral surgeons (S.K. and M.K) with more than 10 years of professional experience who performed the maxillary sinus augmentations. The samples were fixed with buffered, paraformaldehyde fixative solution (Roti Histofix $^{\circledR} 4.5 \%$; Carl Roth GmbH + Co KG, Karlsruhe, Germany), and $\mu-\mathrm{CT}$ of the filled snap-lock tubes was performed. 
Bony cylinders were harvested from the grafted areas during implant placement 5 months after maxillary sinus augmentation by means of a trephine bur with an internal diameter of $3.2 \mathrm{~mm}$ (Fig. 1). The cylinders were gently removed from the trephine and immediately immersed in $4.5 \%$ buffered, $\mathrm{pH} 7.0$ paraformaldehyde fixative (Roti Histofix $4.5 \%$; Carl Roth GmbH + Co KG, Karlsruhe, Germany) for at least 7 days. After dehydrating in alcohol in ascending ethanol series, the cylinders were embedded in paraffin according to standard protocols (Hypercenter XP, Shandon, Frankfurt am Main, Germany) and a $\mu$-CT examination was performed.

A total of 46 samples were evaluated (23 samples of the particulated graft maintained at time of sinus augmentation and 23 bony samples retrieved 5 months after healing). Seven patients were consecutively excluded from the study: one of group A, two of group B, and two of group $\mathrm{C}$ because of insufficient sample retrieval at re-entry. Two patients were additionally excluded from group $\mathrm{C}$ because of steroid medication after maxillary sinus augmentation.

\section{$\mu-\mathrm{CT}$}

All analyses were carried out using a high-resolution $\mu$-CT with a nominal isotropic resolution of $6 \times 6 \times 6 \mu \mathrm{m}^{3}$ voxelsize (SCANCO ${ }^{\circledR} \mu-C T$ 40, SCANCO Medical AG, Brüttisellen, Switzerland) at identical settings $\left(70 \mathrm{kV}, 113 \mu \mathrm{A}, 0.18^{\circ}\right.$ angular increment, $300 \mathrm{~ms}$ integration time).

Each polypropylene snap-lock tube containing the particulated bone or bone and substitute material was clamped into a specimen holder of the $\mu$-CT for examination.

The paraffin-embedded samples retrieved 5 months later were placed into conical pipette tips (100 $\mu$ l MBP Pipet Tips No. 3550, Molecular Bioproducts, Inc., USA), such that the tips held the samples through an interference fit. The pipette was then fixed in a specimen holder of the $\mu$-CT for examination avoiding movements.

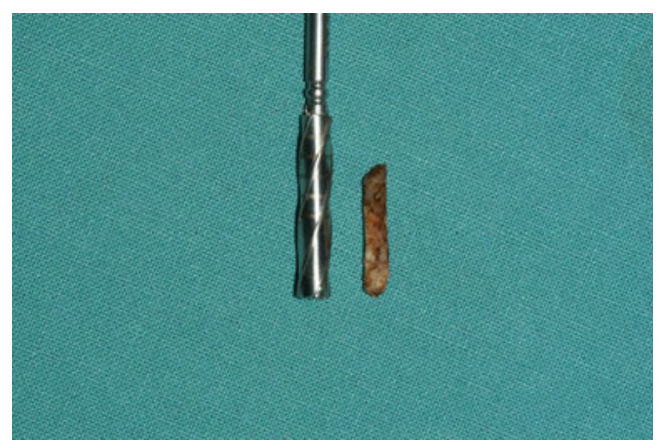

Fig. 1 Five months after maxillary sinus augmentation, samples of the augmented areas were retrieved by means of a trephine bur and embedded in paraffine before $\mu$-CT analyses
Three-dimensional reconstructions of the $\mu$-CT data were performed (Figs. 2 and 3) based on thresholds for bone and substitute material according to histogram distribution of grey values described in a previous study of Kühl et al. [28]. The thresholds for bone were set at 280-550 $\mathrm{mgHA} / \mathrm{cm}^{3}$ (representing the intermediatedensity material) and at $550-1,000 \mathrm{mgHA} / \mathrm{cm}^{3}$ (representing high-density material) for the BSM. Following the segmentation process, a morphological filter operation by means of dilatation and erosion in the border zones of the substitute materials was applied prior to the evaluation with the SCANCO imaging software in order to reduce artefacts. For volume analysis, a volume of interest was first determined, in which the alveolar bone was not included. Based on the segmentation process, the volume of bone $(\mathrm{Vb})$ and the volume of BSM (Vsm) were separately evaluated within the volume of interest. Percentage distribution of bone (Vfb) and BSM (Vfsm) were calculated as volume fractions by dividing $\mathrm{Vb}$ or $\mathrm{Vsm}$ by the volume of interest.

\section{Statistical analyses}

The mean percentage volume fraction of bone $(\mathrm{Vfb})$ and BSM (Vfsm), including standard deviation (SD), were calculated for each group at the time of maxillary sinus augmentation and again 5 months later. An analysis of variance was performed to evaluate statistically significant differences between the individual groups with regard to the changes of $\mathrm{Vfb}$ and $\mathrm{Vfsm}$ between the two points in time (at time of maxillary sinus augmentation and 5 months later) and between each group. Significance was considered to be $p<0.05$. The statistical analyses were performed with a statistics package (SPSS).

\section{Results}

The Vfsm decreased in both groups 5 months after sinus augmentation (Table 1) from a mean Vfsm of $8.9 \%$ to $5.1 \%$ in group A and from $10.1 \%$ to $5.0 \%$ in group B (Fig. 4). Based on the data obtained, a power analysis was performed showing a level of $<80 \%$ for both groups.

In contrast to this, the Vfb showed a statistically significant increase 5 months after maxillary sinus augmentation in all groups (Table 2), whereas the Vfb increased by a mean of $17.1 \%$ in group $\mathrm{A}$, by a mean of $21.7 \%$ in group $\mathrm{B}$, and by $18.5 \%$ in group C (Fig. 5). The increase of bone between the groups showed no statistically significant differences at $p>0.05$ (Table 2). The power analysis showed a level of significance $>80 \%$. 

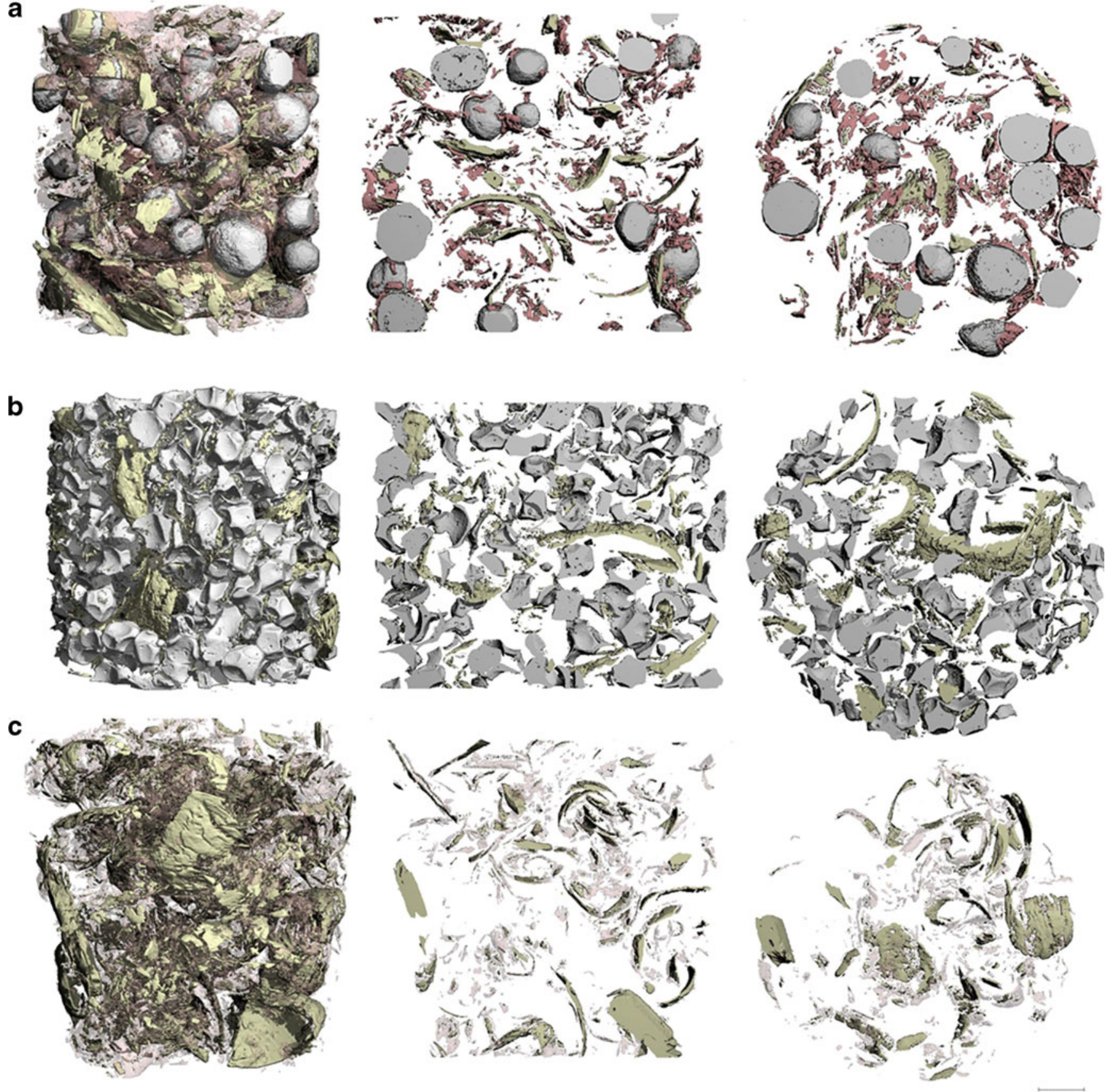

Fig. 2 3D reconstructions after segmentation of the $\mu$-CT data for a sample maintained at the time of maxillary sinus augmentation of group A (upper row) B (middle row), and C (lower row). The bone is green and the BSM particles are gray. The left image shows the

\section{Discussion}

The volume distribution of bone, the bone density, and the bone stability stand in close relationship one to another. BSM has no mechanical stability in its particulated form. To gain biomechanical stability, it has to be surrounded by bone: the greater the bone volume, the higher the strength will be. The volume of newly formed bone is therefore an structure of the grafts' total volume at the time of maxillary sinus augmentation; the middle and right images, respectively, show longitudinal and cross-sectional slices through the particulated grafts

important aspect in comparing different grafting materials. Beside volume, the bone quality plays a key role in biomechanical stability. The bone quality is generally determined by evaluating the bone's density. Bone stability grows with increasing density. Taking this into account, it is of special clinical interest to evaluate both volume and density of the bone obtained after grafting with different materials. The current investigation using $\mu$-CT revealed a statistically 
Fig. 3 3D reconstructions after segmentation of the $\mu$-CT data of a sample of group A (upper row), group B (middle row), and $\mathrm{C}$ (lower row) retrieved 5 months after maxillary sinus augmentation. The bone is green and the BSM is gray. The volume of the bone and the BSM were calculated according to the segmentation a

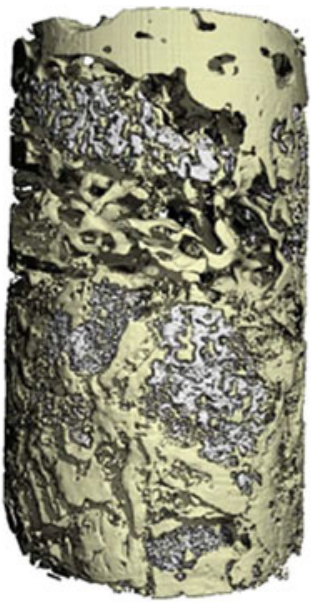

b

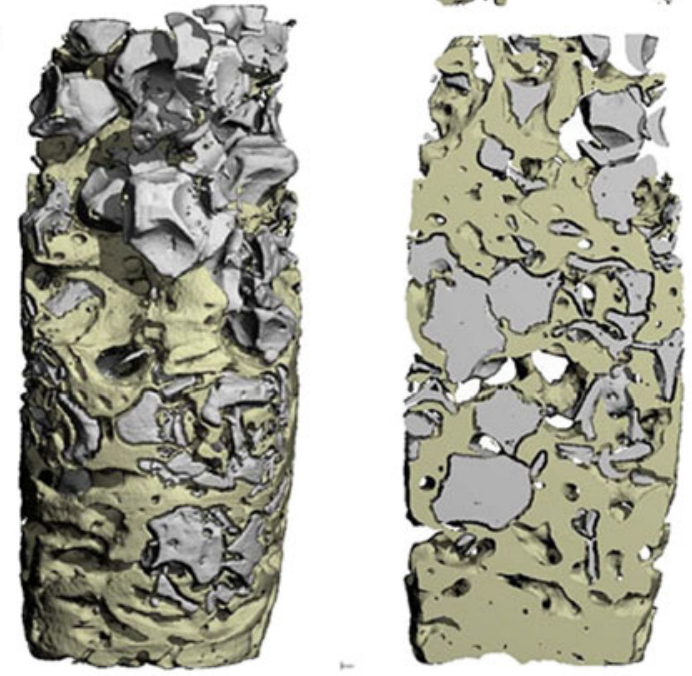

C
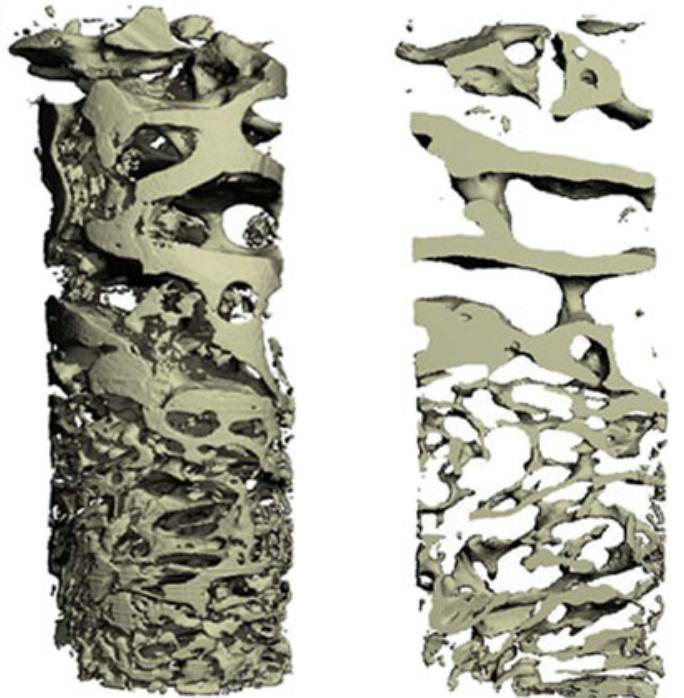
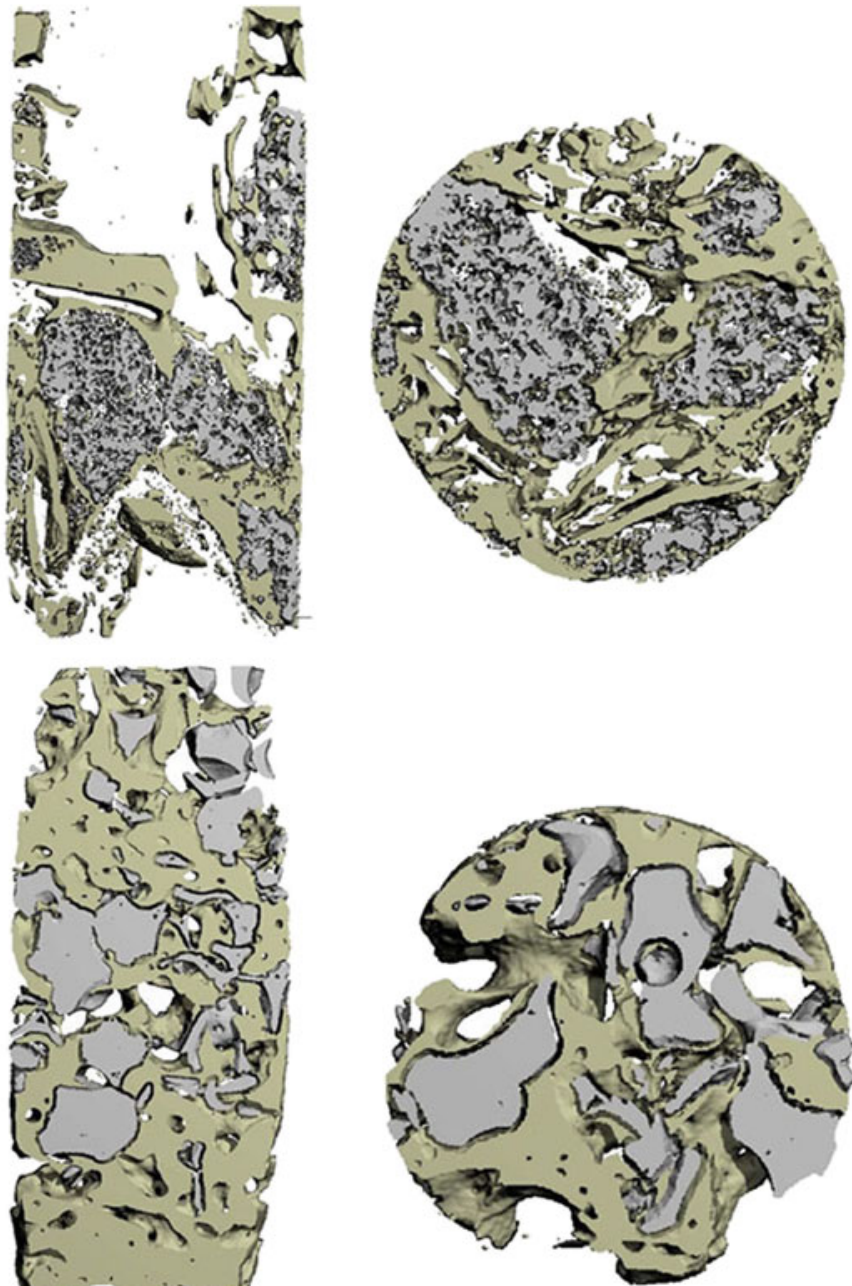

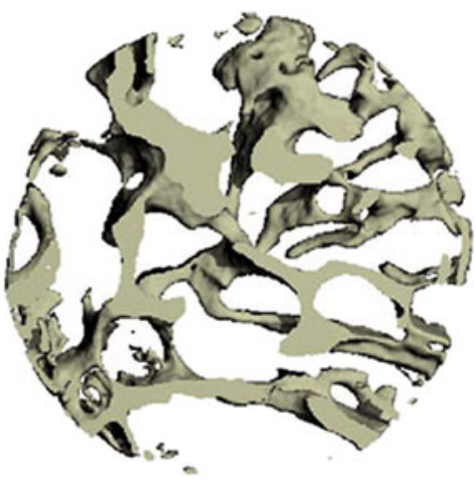

significant increase in the percentage of bone 5 months after maxillary sinus augmentation for three different grafting materials. Autogenous bone is regarded as standard for grafting procedures because of its osteoinductive properties $[23,26,27]$. It was therefore expected that the amount of newly formed bone might be negatively affected by adding bone substitute material particles providing osteoconductive properties only. Frenken et al. [25] used a mixture of HA/ $\beta$ TCP (Straumann ${ }^{\circledR}$ Bone Ceramic) without adding autogenous bone for maxillary sinus augmentation. After an 
Table 1 Percentage volume of bone substitute materials (Vfsm) for both groups: $\mathrm{A}=$ autogenous bone $+\beta-\mathrm{TCP}$ and $\mathrm{B}=$ autogenous bone $+\beta$ $\mathrm{TCP} / \mathrm{HA}$ at different stages $(\mathrm{t} 0=$ at time of maxillary sinus augmentation; $\mathrm{t} 1=5$ months after maxillary sinus augmentation) (number of samples $(N)$, minimum, maximum, mean and standard deviation (SD))

\begin{tabular}{|c|c|c|c|c|c|c|}
\hline \multicolumn{7}{|c|}{ Vfsm $(\%)$} \\
\hline \multicolumn{2}{|c|}{ Groups } & \multirow{2}{*}{$\frac{N}{9}$} & \multirow{2}{*}{$\frac{\text { Min. }}{2.2}$} & \multirow{2}{*}{$\frac{\text { Max. }}{17.7}$} & \multirow{2}{*}{$\frac{\text { Mean }}{8.9}$} & \multirow{2}{*}{$\frac{\mathrm{SD}}{5.4}$} \\
\hline A & t0 & & & & & \\
\hline & t5 & 9 & 0.1 & 16.5 & 5.1 & 5.9 \\
\hline \multirow[t]{2}{*}{ B } & t0 & 8 & 6.5 & 15.3 & 10.1 & 3.2 \\
\hline & $\mathrm{t} 5$ & 8 & 0.7 & 13.3 & 5.0 & 4.1 \\
\hline
\end{tabular}

average healing time of 6 months, the ratio bone volume to total volume was measured to be $27.3 \%$ in histological samples. As no autogenous bone was used in this study, the newly formed bone represents the effective bone volume increase 6 months after maxillary sinus augmentation. In contrast to this, Cordaro et al. [18], in a similar study, found a rate of newly formed bone of $19.8 \%$ with the same $\mathrm{HA} / \beta$ TCP (Straumann Bone Ceramic ${ }^{\circledR}$ ) substitute material. As it is known that autogenous bone has osteoinductive properties, the combination of autogenous bone and substitute materials was expected to be beneficial with regard to the obtained volume of newly formed bone in our study. In our study, however, the effective volume of newly formed bone -i.e., the difference between the bone volume within the grafts at the time of sinuslift procedure and the samples retrieved 5 months later-was measured to be $21.7 \%$. This indicates a lower bone formation rate than in the study published by Frenken et al. and a slightly higher value than in the study of Cordaro et al. $[18,25]$. The reason for this discrepancy might be related to the different mean healing time of approximately 4 weeks between the studies with the shortest healing times for the study of Cordaro et al. [18]. Artzi et al. [29] found that the percentage amount of newly formed bone increases over an extended healing period. In their study, autogenous bone and HA/ $\beta$-TCP were mixed at a ratio of $1: 1$, and bony samples were retrieved 6 and 9 months after grafting the maxillary sinus. The amount of newly formed bone increased from $28.6 \%$ after 6 months to $41.6 \%$ after an average healing time of 9 months. Based on these observations, it can be assumed that the measured $\mathrm{Vfb}$ in our study would also have been higher than $24.8 \%$ with a longer healing period.

The bone volume ingrowth of $17.1 \%$ for group $\mathrm{A}(\beta-\mathrm{TCP}$, Cerasorb $^{\circledR}$ ) was the smallest, resulting in a $\mathrm{Vfb}$ of $21.4 \%$. Artzi et al. [19] used a mixture of $\beta$-TCP $\left(\right.$ Cerasorb $\left.^{\circledR}\right)$ and autogenous bone in a clinical study for maxillary sinus augmentation and found an average bone area fraction of $32 \%$ after a healing time of 12 months. This difference to our study may also be related to the longer healing time.

Suba et al. [22] performed a study on bilateral sinuslift, using autogenous particulated bone on one side and $\beta$-TCP $\left(\right.$ Cerasorb $\left.^{\circledR}\right)$ on the other. In contrast to the above findings, the trabecular bone volume was $32.4 \%$ for the experimental side $\left(\right.$ Cerasorb $\left.^{\circledR}\right)$ and $34.7 \%$ for the control sides in samples retrieved 6 months after sinus augmentation. As observed in

Fig. 4 Mean differences between Vfsm at time of maxillary sinus augmentation and 5 months later for group $\mathrm{A}$ (left boxplot) and group B (right boxplot). The differences between the two groups (A and B) were not statistically significant $(p=0.728)$

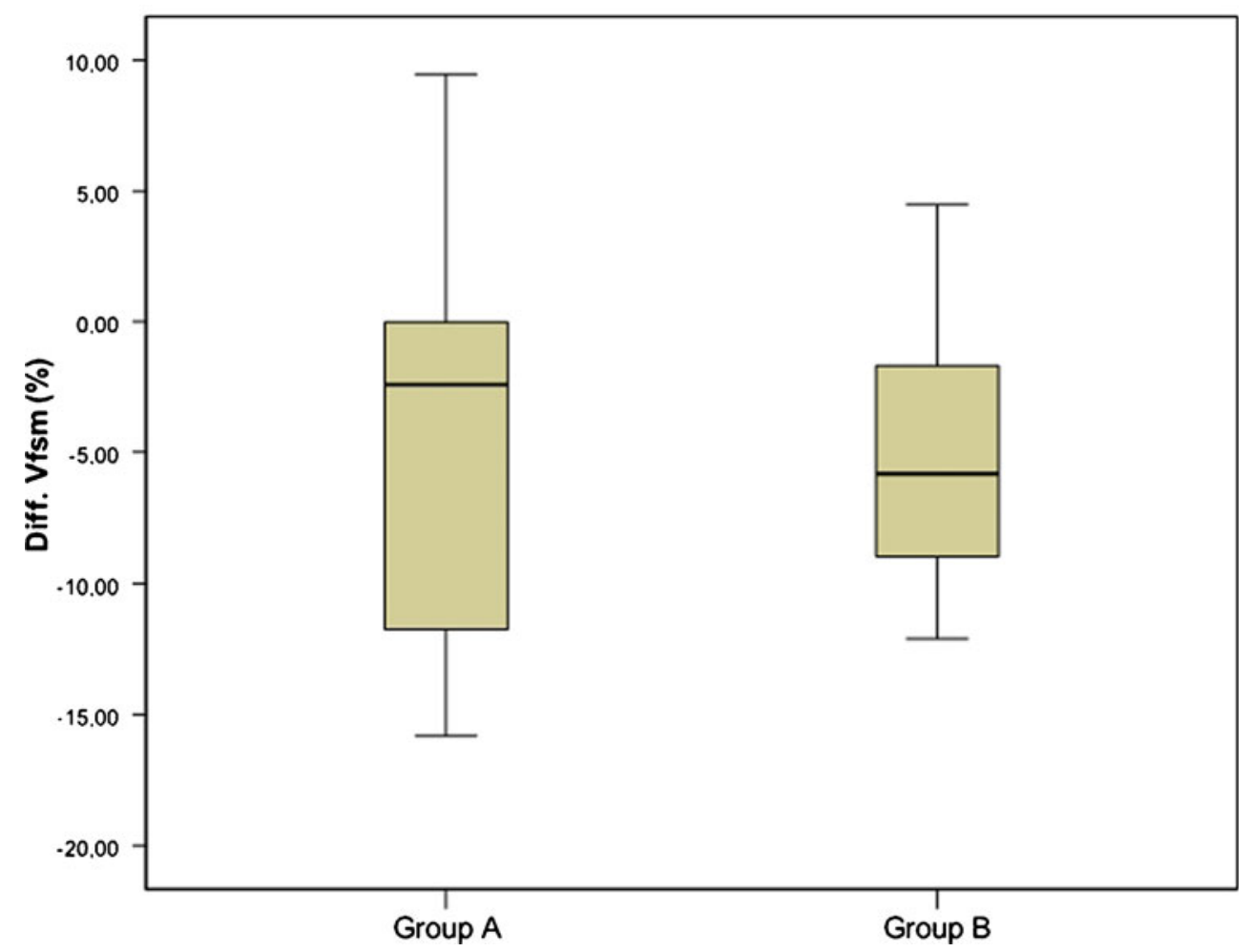


Table 2 Percentage volume of bone (Vfb) for all groups: $\mathrm{A}=$ autogenous bone $+\beta$-TCP, $\mathrm{B}=$ autogenous bone $+\beta$-TCP/HA and $\mathrm{C}=$ autogenous bone at different stages $(\mathrm{t} 0=$ at time of maxillary sinus augmentation; $\mathrm{t} 1=5$ months after maxillary sinus augmentation) (number of samples $(N)$, minimum, maximum, mean and standard deviation (SD))

\begin{tabular}{|c|c|c|c|c|c|c|c|c|c|}
\hline \multicolumn{8}{|l|}{$\mathrm{Vfb}(\%)$} & \multirow[b]{2}{*}{ Diff. Vfb (\%) } & \multirow[b]{2}{*}{$p$ (Diff. Vfb) } \\
\hline Groups & & $N$ & Min. & Max. & Mean & SD & $p$ & & \\
\hline \multirow[t]{2}{*}{ A } & to & 9 & 2.4 & 6.3 & 4.3 & 1.2 & \multirow[t]{2}{*}{0.01} & \multirow[t]{2}{*}{17.1} & \multirow[t]{6}{*}{0.578} \\
\hline & t5 & 9 & 4.5 & 34.3 & 21.4 & 9.0 & & & \\
\hline \multirow[t]{2}{*}{ B } & to & 8 & 1.6 & 7.0 & 3.1 & 1.8 & \multirow[t]{2}{*}{0.01} & \multirow[t]{2}{*}{21.7} & \\
\hline & $\mathrm{t} 5$ & 8 & 16.2 & 42.0 & 24.8 & 10.2 & & & \\
\hline \multirow[t]{2}{*}{$\mathrm{C}$} & to & 6 & 3.1 & 13.1 & 7.0 & 3.4 & \multirow[t]{2}{*}{0.03} & \multirow[t]{2}{*}{18.5} & \\
\hline & $\mathrm{t} 5$ & 6 & 15.9 & 31.6 & 25.5 & 5.7 & & & \\
\hline
\end{tabular}

$P$ values indicate statistical significance with $p<0.05$ within each group between t 0 and $\mathrm{t} 1$. Diff. Vfb represents the mean difference of bone volume between $\mathrm{t} 0$ and t1. $P$ value for diff. Vfb indicates no statistically significant differences between the groups

our study, the bone volume ingrowth was smaller for the $\beta$ TCP group than for the control group with autogenous bone alone.

In comparison to groups $\mathrm{A}$ and $\mathrm{B}$, the bone ingrowth in group $\mathrm{C}$ (autogenous bone alone) was measured to be $18.5 \%$, resulting in a bone $\mathrm{Vfb}$ of $25.53 \%$, which represents the highest bone volume to total volume value of the three groups. The higher amount of bone volume might be explained by the higher $\mathrm{Vfb}$ of $7.0 \%$ at the time of maxillary sinuslift in comparison to group A $(4.3 \%)$ and group B $(3.1 \%)$. Taking this into consideration, the effective bone volume ingrowth of $18.5 \%$ ranges between the effective bone ingrowth of groups A $(17.1 \%)$ and $B(21.7 \%)$.
The data revealed that there is no statistically significant difference in Vf in any of the groups. The reason for the same ingrowth in all groups cannot be determined by this study. Obviously in the present clinical setup, BSM showed sufficient osteogenic potential to support similar bone ingrowth as seen in controls. In agreement with data from other studies, our findings seem to support clinical application of BSM for sinus grafting procedures. Additionally, BSM have shown less tendency for resorption in clinical long-term applications compared to autogenous bone grafts and may potentially be beneficial with regard to donor site, harvest morbidity, and patients' acceptance.

Our data indicate that using mixtures of autogenous bone and $\beta$-TCP or mixtures of PAB and HA/ $\beta-$ TCP as well as

Fig. 5 Mean differences between $\mathrm{Vfb}$ at time of maxillary sinus augmentation and 5 months later for group A (left boxplot), group B (middle boxplot), and group $\mathrm{C}$ (right boxplot). There was no statistically significant difference in bone increase between the three groups $(p=0.578)$. Outlayer appeared only in group $\mathrm{A}$ and $\mathrm{B}$ with more than $30 \%$ of newly formed bone in group $\mathrm{C}$ and a negative difference in on case of group B due to bony resorption

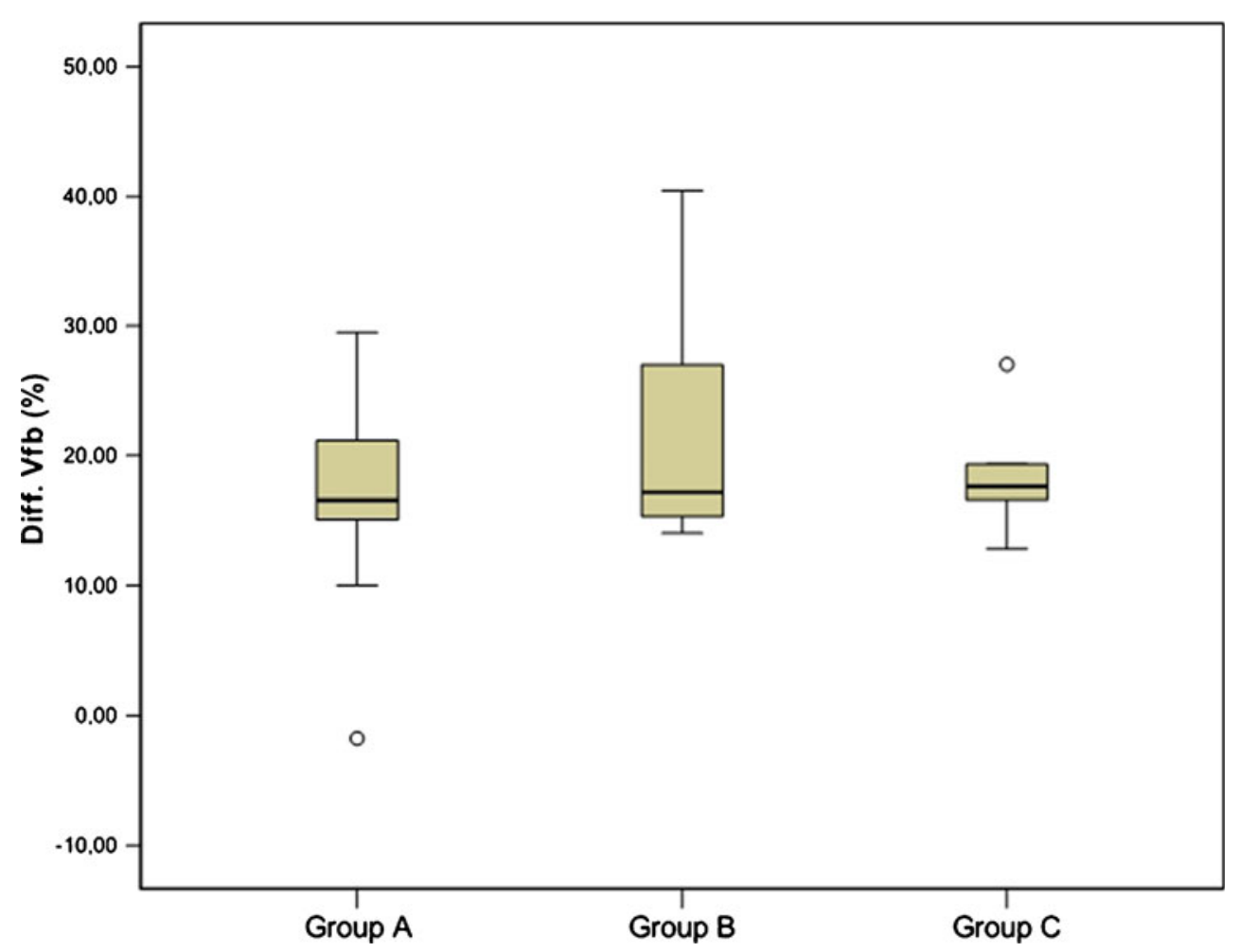


PAB alone for maxillary sinuslift procedure result in a remarkable amount of newly formed bone after a healing time of 5 months. Differences between the investigated materials are negligible, and it can be assumed that these differences are of limited clinical importance. However, the volume of newly formed bone in our study was not negatively affected by adding BSM to autogenous bone. Adding BSM to PAB may reduce donor site morbidity since less autogenous material is needed. This is of clinical relevance in cases with severe atrophy of the jaws.

Acknowledgments The study was partly funded by a study grant of the University of Mainz (MAIFOR). We extend our gratitude to Prof. Dr. Heinz Duschner for the facilities in terms of $\mu$-CT acquisition and Irene Mischak for the statistical analysis.

Conflict of interests The authors declare that they have no conflict of interests.

\section{References}

1. Boyne PJ, James RA (1980) Grafting of the maxillary sinus floor with autogenous marrow and bone. Int J Oral Maxillofac Surg 38:613-661

2. Tatum OH (1986) Maxillary and sinus implant reconstruction. Dent Clin N Am 30:207-229

3. Huerzeler MB, Kirsch A, Ackermann KL, Quinones CR (1996) Reconstruction of the severely resorbed maxilla with dental implants in the augmented maxillary sinus: a 5-year clinical investigation. Int $\mathrm{J}$ Oral Maxillofac Implants 11:466475

4. Wallace ST, Froum ST (2003) Effect of maxillary sinus augmentation on the survival of endosseous dental implants. A systematic review. Ann Periodontol 8:328-343

5. Kreisler M, Moritz O, d'Hoedt B (2006) Evidence-based medicine in sinus floor elevation part 1: general aspects and the influence of the grafting material on implant prognosis. J Dent Implantol 22:299-323

6. Kreisler M, Moritz O, d'Hoedt B (2007) Evidence-based medicine in sinus floor elevation part 2: direct and indirect factors in sinus floor elevation and their influence on implant prognosis. J Dent Implantol 23:68-86

7. Froum SJ, Tarnow DP, Wallace SS, Rohrer MD, Cho SC (1998) Sinus floor elevation using bovine bone mineral (OsteoGraf/N) with and without autogenous bone: a clinical, histologic, radiographic and histomorphometric analysis-part 2 of an ongoing prospective study. Int $\mathrm{J}$ Periodonics Restor Dent 18:528-543

8. Hallman M, Sennerby L, Lundgren S (2002) A clinical and histologic evaluation of implant integration in the posterior maxilla after sinus floor augmentation with autogenous bone, bovine hydroxyapatite, or a 20:80 mixture. Int J Oral Maxillofac Implants 17:635643

9. Schlegel KA, Fichtner G, Schultze-Mosgau S, Wiltfang J (2003) Histologic findings in sinus augmentation with autogenous bone chips versus a bovine substitute material. Int J Oral Maxillofac Implants 18:53-58

10. Hatano N, Shimizu Y, Ooya K (2004) A clinical long-term radiographic evaluation of graft height changes after maxillary sinus floor augmentation with a 2:1 autogenous/xenograft mixture and simultaneous placement of dental implants. Clin Oral Implants Res 15:339-345

11. Guarnieri R, Grassi R, Ripari M, Pecora G (2006) Maxillary sinus augmentation using granular calcium sulfate (surgiplaster sinus): radiographic and histologic study at 2 years. Int $\mathrm{J}$ Periodontics Restorative Dent 26:79-85

12. Wanschitz F, Figl M, Wagner A, Ewers R (2006) Measurement of volume changes after sinus floor augmentation with phycogenic hydroxyapatite. Int $\mathrm{J}$ Oral Maxillofac Implants 21:422-438

13. Johansson LA, Isaksson S, Lindh C, Becktor JP, Sennerby L (2010) Maxillary sinus floor augmentation and simultaneous implant placement using locally harvested autogenous bone chips and bone debris: a prospective clinical study. Int J Oral Maxillofac Implants 68:837-844

14. Yildirim M, Spiekermann H, Biesterfeld S, Edelhoff D (2000) Maxillary sinus augmentation using xenogenic bone substitute material Bio-Oss ${ }^{\circledR}$ in combination with venous blood. A histologic and histomorphometric study in humans. Clin Oral Implants Res $11: 217-229$

15. Maiorana C, Sigurta D, Mirandola A, Garlini G, Santoro F (2006) Sinus elevation with alloplast or xenogenic materials and implants: an up-to-4-year clinical and radiologic follow-up. Int J Oral Maxillofac Implants 21:426-432

16. Esposito M, Grusovin MG, Rees J, Karasoulos D, Felice P, Alissa $\mathrm{R}$ et al (2010) Interventions for replacing missing teeth: augmentation procedures of the maxillary sinus. Cochrane Database Syst Rev 3:CD008397

17. Froum SJ, Wallace SS, Cho SC, Elian N, Tarnow DP (2008) Histomorphometric comparison of a biphasic bone ceramic to anorganic bovine bone for sinus augmentation: 6- to 8-month postsurgical assessment of vital bone formation. A pilot study. Int J Periodontics Restorative Dent 28:273-281

18. Cordaro L, Bosshardt DD, Palattella P, Rao W, Serino G, Chiapasco M (2008) Maxillary sinus grafting with Bio-Oss ${ }^{\circledR}$ or Straumann ${ }^{\circledR}$ Bone Ceramic: histomorphometric results from a randomized controlled multicenter clinical trial. Clin Oral Implants Res 19:796-803

19. Artzi Z, Kozlovsky A, Nemcovsky CE, Weinreb M (2005) The amount of newly formed bone in sinus grafting procedures depends on tissue depth as well as the type and residual amount of the grafted material. J Clin Periodontol 32:193-199

20. Huerzeler MB, Quinones CR, Kirsch A, Schupbach P, Krausse A, Strub JC et al (1997) Maxillary sinus augmentation using different grafting materials and dental implants in monkeys. Part III. Evaluation of autogenous bone combined with porous hydroxyapatite. Clin Oral Implants Res 8:401-411

21. Scarano A, Degidi M, Iezzi G, Pecora G, Pattelli M, Orsini G et al (2006) Maxillary sinus augmentation with different biomaterials: a comparative histologic and histomorphometric study in man. Implant Dent 15:197-207

22. Suba Z, Takacs D, Matusovits D, Barabas J, Fazekas A, Szabo G (2006) Maxillary sinus floor grafting with B-tricalcium phosphate in humans: density and microarchitecture of the newly formed bone. Clin Oral Implants Res 17:102-108

23. Burchardt H (1983) The biology of bone graft repair. Clin Orthop Relat Res 174:28-42

24. van den Bergh JP, ten Bruggenkate CM, Krekeler G, Tuinzing DB (1998) Sinusfloor elevation and grafting with autogenous iliac crest bone. Clin Oral Implants Res 9:429-435

25. Frenken JWFH, Bouwman WF, Bravenboer N, Zijderveld SA, Schulten EAJM, Bruggenkate CM (2010) The use of Straumann ${ }^{\circledR}$ Bone Ceramic in maxillary sinus floor elevation procedure: a clinical, radiological, histological and histomorphometric evaluation with a 6month healing period. Clin Oral Implants Res 21:201-208 
26. Jensen OT, Shulman LB, Block MS, Iacono VJ (1998) Report of the Sinus Consensus Conference of 1996. Int J Oral Maxillofac Implants 13:11-45

27. Tong DC, Rioux K, Drangsholt M, Beirne OR (1998) A review of survival rates for implants placed in grafted maxillary sinuses using metaanalysis. Int J Oral Maxillofac Implants 13:175-182

28. Kühl S, Götz H, Hansen T, Kreisler M, Behneke A, Heil U et al (2010) Three-dimensional analysis of bone formation after maxillary sinus augmentation by means of microcomputed tomography: a pilot study. Int J Oral Maxillofac Implants 25:930-938

29. Artzi Z, Weinreb M, Carmeli G, Lev-Dor R, Dard M, Nemcovsky CE (2010) Histomorphometric assessment of bone formation in sinus augmentation utilizing a combination of autogenous and hydroxyapatite/biphasic tricalcium phosphate graft materials: at 6 and 9 month in humans. Clin Oral Implants Res $19: 686-692$ 